\title{
Hemoglobina glicosilada, colesterol y tensión arterial: una manera de predecir nefropatía diabética en diabéticos tipo II
}

Main Risk Factors for Nephropathy in Type 2 Diabetes Mellitus are Plasma Cholesterol Levels, mean Blood Pressure, and Hyperglycemia. Ravid M; Brosh D; Ravid -Safran D. Arch. Intern. Med. 1998 May 11: 158: 998-1004.

\section{Objetivo}

Determinar predictores de riesgo de desarrollo y progresión de nefropatía diabética (ND) en pacientes diabéticos no insulinodependientes (DBT II).

\section{Diseño}

Estudio de cohorte prospectivo.

\section{Lugar}

Tel Aviv. Israel.

\section{Pacientes}

Se incluyeron 574 pacientes entre 40 a 60 años con menos de 5 años del diagnóstico de DBT II y creatinina sérica $(\mathrm{Cr})<1.4 \mathrm{mg} \%$, tensión arterial (TA) < a 140/90, albuminuria de 24 horas (Alb24) $<30 \mathrm{mg} /$ $24 \mathrm{hs}$ e índice de masa corporal (IMC) $<35$. El tiempo medio de seguimiento fue $7.8 \pm 0.9$ años.

Los pacientes recibían como tratamiento al inicio del estudio: insulina $(n=62)$, hipoglucemiantes orales (HGO) $(n=235)$ y dieta $(n=324)$.

\section{Evaluación de factores pronósticos}

Se evaluó Hemoglobina glicosilada ( $\mathrm{HbA}), \mathrm{Cr}$, Alb24 y TA semestralmente. El colesterol e IMC se registraron anualmente.

Los pacientes hipertensos recibieron bloqueantes cálcicos o tiazidas como tratamiento.

Los percentilos 50 de las 3 principales características fueron los siguientes: HbA: 9\%, TA media: $95 \mathrm{mmHg}$ y colesterol: $203 \mathrm{mg} \%$. Cuando las 3 variables daban un percentilo $\geq 50$ se consideraba población de alto riesgo (PAR) y < 50 población de bajo riesgo (PBR)

\section{Medición de resultados principales}

Se evaluó como resultado primario la Alb24 y Cr. Otros resultados evaluados fueron eventos clínicos: muerte, infarto agudo de miocardio no fatal, angor, insuficiencia cardíaca congestiva y enfermedad vascular periférica.

\section{Resultados Principales}

De los 574 pacientes, 373 (65\%) tuvieron Alb24 normal al final del seguimiento, 201 (35\%) desarrollaron microalbuminuria (30-300 $\mathrm{mg} / 24$ hs.) 90 de los cuales (16\%) progresaron a macroalbuminuria (> $300 \mathrm{mg} . / 24 \mathrm{hs}$.). El análisis multivariable para evaluar factores de riesgo independiente indicó que el colesterol, la TA media y la HbA fueron los principales predictores de nefropatía diabética. Comparando la PAR con la PBR, el Odds Ratio (OR) para desarrollo de microalbuminuria fue 43 (IC 95\% 25-106). Para progresión a macroalbuminuria el OR fue 18 (IC 95\% 11-33). Para eventos clínicos combinados el OR fue 15 (IC 95\% 9-25). El colesterol HDL, el IMC, el tabaquismo, el nivel socioeconómico bajo y el sexo masculino fueron los otros predictores significativos de nefropatía diabética.

Al realizar un análisis de los distintos subgrupos acordes al tratamiento inicial (insulina, $\mathrm{HGO}$, dieta), éste no predijo desarrollo de nefropatía. Los pacientes de la PAR tuvieron más riesgo de tener eventos clínicos o fallecer durante el seguimiento $O R=14,75$ (IC 95\% 8.72-24.95; $\mathrm{P}=0.001$ ) comparando con PBR.

\section{Conclusiones}

La combinación de los valores de TA en el rango normal alto, con moderada elevación de colesterol y HbA define una población de alto riesgo para el desarrollo de nefropatía diaética y eventos clínicos relacionados a la arteriosclerosis.

\section{COMENTARIO}

El presente trabajo es uno más, de los tantos estudios epidemiológicos que corrobora que la hiperglucemia es un:predictor de desarrollo de complicaciones microangiopáticas (en este caso nefropatía diabética). Probablemente su precedente más contundente es el trabajo de Wisconsin ${ }^{1}$. La hipertensión arterial y la hipercolesterolemia también han sido reportadas como predictores de nefropatía diabética, y más aún, se sabe que el adecuado control de TA retrasa el desarrollo de la misma y que el control del colesterol retrasa las complicaciones macroangiopáticas en los DBT II ${ }^{2}$. El efecto del control de la glucemia en DBT II y la progresión de nefropatía es más controvertido. El presente trabajo, es un estudio observacional y no esta diseñado para evaluar el efecto de las intervenciones, sino para evaluar el pronóstico en un grupo de pacientes con DBT II. Por lo tanto, de este estudio no puede concluirse que bajar la glucemia sea beneficioso para prevenir nefropatia diabética (aunque intuitivamente se crea que sí).

Existen antecedentes de trabajos experimentales que parecieran de- mostrar que el descenso de la glucemia retrasa el desarrollo de la nefropatía diabética. El estudio de los veteranos ${ }^{3}$ y un estudio Japonés 4 mostraron resultados positivos mientras que otro estudio previo no lo había podido demostrar ${ }^{5}$. (Ver Evidencia Nro. 4 - 1998 pág. 15)

El presente trabajo no esta diseñado para resolver este interrogante. Lo que sí nos aporta, son básicamente 3 elementos:

1- Discrimina sencillamente una población de alto riesgo para el desarrollo de ND y EC en quienes deberíamos extremar los controles de glucemia y factores de riesgo asociados.

2- El desarrollo de ND dependió de los valores de estos predictores independientemente del tratamiento realizado (dieta, $\mathrm{HO}$, insulina). 3.-El punto de corte de TA tomado para ser considerado PAR, estuvo por debajo de los valores de TA normal-alta $(<130-85)$. Por lo que se concluye, en coincidencia con la recomendación del VI Reporte del Comité Americano sobre prevención, detección, evaluación y tratamiento de la HTA $^{6}$, que los diabéticos deberían manejar cifras de TA $<130 / 85$ para reducir el riesgo de eventos micro y macrovasculares.

. Klein R Hyperglicemia and microvascular and macrovascular disease in diabetes. Diaberes Care. 1995:18: 258-268.

2. Sawicki PT, Pharmacological treatment of diabetes patients with cardiovascular complications. J.Intern.Med. 1998 Mar; 243(3): 181-189.

3. Abraira C.Veterans Affairs Cooperative Study on glycemic control and complications in type II diabetes (VA CSDM). Result of the feasibility trial Diabetes Care. 1995;18:1113-1123

4. Ohkubo Y. Intensive insuline therapy prevents the progression of diabetic microvascular complications in Japanese patiens with non insulin dependent diabetes mellitus: a randomized prospective 6 year

study.Diabetes Res Clin Pract.1995; 28:103-117.

5. University Group Diabetes program. Effects of hypoglycemic agents on vascular complications in patients with adult onset diabetes. Diabetes. 1982;31:1-81.

6. The sith report of the Joint national Committee on Prevention, Detection, Evaluation and Treatment of High Blood Pressure. Arch Intern Med 1997; 157: $2431-2445$. 